\title{
Leucemia Mielóide Crônica - Transplante de medula óssea
}

\section{Cronic Myeloid Leukemia - Bone marrow transplantation}

Francisco J. P. Aranha

\section{Introdução}

A Leucemia Mielóide Crônica (LMC) foi descrita pela primeira vez no início do século 19. Até 1960 pouco se compreendia da biologia da LMC, quando foi descrita, por Nowell, ${ }^{1}$ o cromossomo Philadelphia, e que este era devido à t(9;22), e a LMC tinha origem de uma única célula progenitora hematopoética; na era da biologia molecular, descreveu-se a fusão do gene BCR-ABL. ${ }^{2,3}$

O tratamento até os anos 80 era insatisfatório. No início tratava-se com arsênico e radioterapia, que tinham um certo controle sobre os sintomas. Nos anos 50, o tratamento de escolha era o bussulfano, com melhora da qualidade de vida, mas também sem impacto na sobrevida global dos pacientes, e havia uma suspeita que os agentes alquilantes poderiam predispor a progressão para uma fase avançada da doença. Então foi substituído pela hidroxicarbamida (Hydrea ${ }^{\circledR}$ ). O primeiro agente com o qual se conseguiu uma remissão citogenética foi com o interferon- $\alpha$. Entre 19802000, para os pacientes que não eram elegíveis ao Transplante de Células Hematopoéticas Progenitoras (TCHP) este era o tratamento de escolha, e com o TCHP a meta era a cura da LMC, com remissão molecular. Com o interferon- $\alpha$, em torno de $60 \%$ dos pacientes alcançavam uma resposta citogenética maior, menor que 35\% das células eram Philadelphia positivas, 89\% dos pacientes em 18 meses estavam vivos em fase crônica. ${ }^{1,2,3}$

Na porta do século 21, ou melhor, em 1998, o tratamento de primeira linha da LMC é com inibidor da tirosina quinase, o primeiro inibidor da tirosina quinase a chegar ao mercado foi o imatinibe. Tanto com o TCHP como com o imatinibe o alvo do tratamento é a remissão molecular. ${ }^{4}$

Portanto, o TCHP, com altas doses de quimioterapia, era o tratamento de escolha para pacientes com diagnóstico de LMC em primeira fase crônica em pacientes jovens, ou seja, menores que 50 anos com doador HLA totalmente compatível, tanto relacionado como não relacionado. Nos anos 90 era o tratamento de escolha, a despeito da morbidade e mortalidade relacionadas ao procedimento (MRP). ${ }^{4,5}$

O papel do TCHP autólogo não existe mais atualmente. Segundo, há uma queda do número de TCHP para LMC em $1^{\text {a }}$ fase crônica, a partir de 2000, com a introdução do imatinibe. Em terceiro que o TCHP para não $1^{\text {a }}$ fase crônica continua em números absolutos constante. ${ }^{7}$

Entre 1980 a 2003 foram realizados 13.416 TCHP para LMC, a mediana de idade foi de 36 anos e $60 \%$ eram do sexo masculino. Houve um aumento entre os doadores não relacionados de $10 \%$ para $36 \%$. A indicação de transplantes em

Médico do Centro de Hematologia e Hemoterapia da Unicamp - Campinas-SP.

Correspondência: Francisco José Penteado Aranha

Universidade Estadual de Campinas - Unicamp

Unidade de TMO HC/Hemocentro - Unicamp - CX Postal: 6198

13083-970 - Campinas-SP - Brasil

E-mail: aranha@unicamp.br 

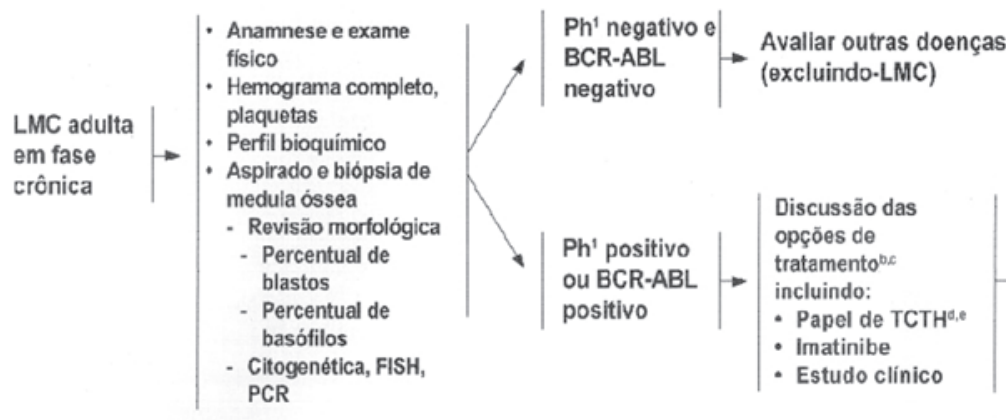

Figura 1. Indicação ao diagnóstico

crise blástica , durante este período, manteve-se estável, com aumento das indicações em segunda fase crônica e/ou em fase avançada da doença, portanto, há aumento da indicação de TCHP de pacientes com maior score de risco do EBMT. A sobrevida é em torno de $60 \%$, com taxa de MRP por volta de $35 \%$ e a recidiva de aproximadamente $20 \%{ }^{7}$

Antes da era imatinibe, antes de 1999, 60\% das indicações do TCHP para LMC era em primeira fase crônica. Pósimatinibe, dados de 2003, a indicação em primeira fase crônica caiu 20\%. Aumento da indicação de TCHP em, pelo menos, fase acelerada ou em segunda fase crônica, houve um aumento de $16 \%$. $^{7}$

\section{Indicação de TCHP}

\section{Ao diagnóstico}

Na era do imatinibe não há indicação de TCHP para LMC em primeira fase crônica. Devemos excetuar os pacientes pediátricos porque não há até o momento um consenso para o uso do imatinibe como primeira linha. Quando não há possibilidade de indicar o uso do imatinibe, tanto por falta de acesso à medicação ou por problemas econômicos, o TCHP está indicado em primeira linha de tratamento. Como conduta, devemos procurar, sempre, um doador HLA totalmente compatível relacionado (Figura 1)

Falha remissão hematológica completa (RHC) em 6 meses

Quando não se consegue uma RHC nos primeiros seis meses de tratamento, que é uma condição rara, acometendo $2 \%$ a $4 \%$ dos pacientes em uso do imatinibe. Deve-se estudar presença de mutações, realizar citogenética tradicional e, quando possível, FISH.

Esta é uma situação em que o TCHP pode ser indicado, tanto com doador relacionado como não relacionado. Pacientes que não tenham doador para realizar TCHP, devese indicar como tratamento uma droga de segunda geração dos inibidores da tirosina quinase. ${ }^{8}$ (Figura 2)

\section{Falha remissão citogenética completa}

em 18 meses

O estudo IRIS mostrou que os resultados são similares para todos pacientes que conseguiram uma RCy completa, independentemente do tempo em que se conseguiu este status. A escolha deste tempo, de 18 meses, como um tempo

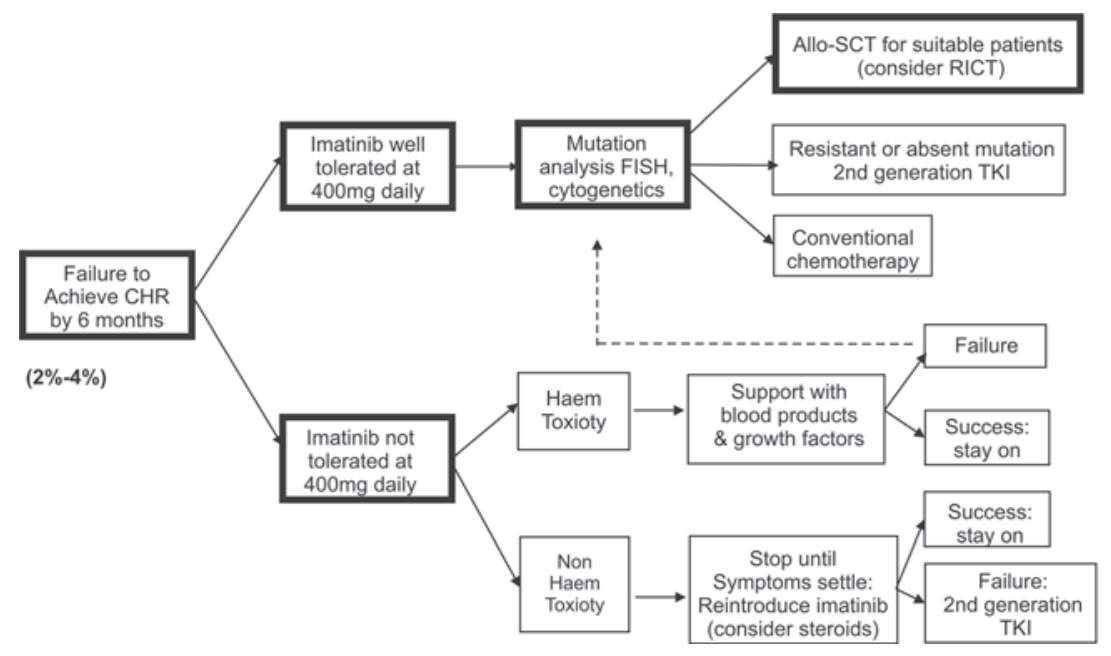

Figura 2. Fluxograma - Falha hematológica após 6 meses de tratamento 
de decisão terapêutica é porque após este período muito pouco pacientes alcançariam uma resposta citogenética maior., ${ }^{8,10}$

Neste panorama deve-se realizar análise das mutações, na ausência de uma mutação continua o tratamento com imatinibe, deve-se aumentar a dose da medicação. ${ }^{8}$

Com presença de mutação, sendo esta sensível a uma droga de segunda geração de inibidor da tirosina quinase é o tratamento de escolha. Quando esta mutação é resistente aos inibidores da tirosina quinase, e o paciente tem um doador HLA compatível, relacionado ou não relacionado, o TCHP está indicado. ${ }^{8}$ (Figura 3)

Perda RHC, RCy completa ou remissão molecular maior (RMM)

O estudo IRIS sugere que pouca diferença, na sobrevida, existe entre pacientes em RCy completa com ou sem RMM. ${ }^{9}$ Tanto para perda RHC e da RCy completa como para a perda RMM devem-se realizar estudos de possíveis mutações, porque a natureza da mutação pode direcionar o tratamento. Se a mutação T315I está presente, e o paciente tem um doador HLA compatível, relacionado ou não relacionado, o TCHP está indicado. ${ }^{8}$

A mutação da região do P-loop apresenta pior sobrevida comparada com as mutações da região não P-loop. E se o paciente tem um doador HLA compatível, relacionado ou não relacionado, o TCHP está indicado, ou também se pode indicar o tratamento com um inibidor da tirosina quinase de segunda geração. ${ }^{8}$ (Figura 4)

\section{Progressão para fase acelerada ou crise blástica}

São duas situações que são incuráveis sem TCHP. Deve-se sempre tentar uma segunda fase crônica, tão logo se tenha alcançado uma segunda FC realizar TCHP.

Para os pacientes que não tenham doador ou possibilidade de submeterem-se a um TCHP, o intuito do tratamento é prolongar a sobrevida do paciente.

\section{Decisões TCHP}

\section{Score}

Os fatores de riscos pré-TCHP para LMC são cinco: tipo de doador, estágio da doença, idade do receptor, combinação sexo entre receptor e doador e, por último, o tempo entre o diagnóstico e o TCHP. Estes fatores de risco são cumulativos para os pacientes com LMC, importantes para uma melhor orientação terapêutica de TCHP. A seleção des- tes fatores de riscos foi baseada em estudos anteriores do grupo europeu, onde estes fatores mostravam-se repetidamente significativos. ${ }^{10-13}$

O risco de um paciente é somente a soma dos fatores de riscos, tipo de doador: (0 doador HLA idêntico relacionado e 1 doador HLA idêntico não relacionado), estágio da doença (0 1a FC, 1 FAe 2 CB/2a FC), idade do receptor ( $0<20$ anos, 1 20-40 anos e $2>40$ anos), combinação de sexo entre receptor e doador (0 para todas as combinações e 1 para receptor masculino com doador feminino). ${ }^{10}$

O score mais baixo é o 0 ; receptores que têm doador HLA compatível e relacionado, o TCHP ocorre no primeiro ano do diagnóstico, em primeira FC, com idade $<20$ anos, e a combinação de sexo entre o receptor e o doador não é de masculino e feminino, com uma probabilidade de sobrevida em cinco anos, global de 76\%, livre de doença de 62\% e mortalidade relacionada ao procedimento de $21 \%$. Ao contrário, o score mais alto possível é o 7 , receptor do sexo masculino, idade acima de 40 anos, em CB, doadora do sexo feminino, HLA compatível não relacionada, com uma probabilidade de sobrevida em cinco anos, global de 19\%, livre de doença de $15 \%$ e mortalidade relacionada ao procedi- 
mento de 81\%. Este sistema de score foi validado por alguns autores. ${ }^{10,14,15}$

Na era dos inibidores da tirosina quinase não há mais dúvidas, pelo menos até este momento, para o tratamento de primeira linha para pacientes com LMC. Mas quando o TCHP está indicado este sistema de score continua de grande valia para estimar a sobrevida global, livre de doença e a mortalidade relacionada ao procedimento.

\section{Doador}

Atualmente com os avanços da metodologia de tipagem HLA, método molecular, melhores cuidados com a terapia de suporte, com as infecções oportunistas e as terapias de imunossupressão mais adequadas, conseguiu-se resultados comparáveis com TCHP com doadores não relacionados em relação aos doadores relacionados, portanto, a escolha de um doador alternativo não é mais um problema a ser levado em conta quando se indica um TCHP. ${ }^{16}$

\section{Condicionamento}

Os esquemas de condicionamento no TCHP, para as doenças malignas, tem o intuito de cura, são regimes de poliquimioterapia mieloablativas. Associado à mieloablação tem-se a ação das células imunocompetentes do enxerto, os linfócitos, sobre o tumor, o efeito denominado como enxerto versus leucemia (EvL). A infusão de linfócitos do doador (ILD), em $60 \%$ a $90 \%$ dos pacientes em FC e $30 \%$ dos pacientes em CB, a remissão completa pode ser restituída, também, por um efeito aloimune. ${ }^{17}$

Por conta deste efeito das células imunocompetentes do enxerto e do ILD à resposta aloimune, introduziu-se o transplante de intensidade reduzida (CIR), em 1998. Desde a introdução desta modalidade de TCHP até 2004 houve um crescimento de 30\% TCHP de intensidade reduzida. Em comparação, ao TCHP mieloablativo, tem maior efeito GVL, a idade limite, dos pacientes, para realização do TCHP de intensidade reduzida são de 10 a 15 anos a mais do que no TCHP mieloablativo. O TCHP CIR apresenta um menor efeito antitumor pela quimioterapia de condicionamento. Em acompanhamento por longo período de tempo, parece que TCHP de RIC pode ter uma maior incidência de recidiva. ${ }^{18,19}$

Até o momento, não há evidências claras que TCHP de CIR seja melhor que TCHP mieloablativas, que ofereça vantagem para pacientes jovens que sejam canditados a um TCHP. Atualmente, é certa a indicação para pacientes idosos ou jovens que não poderia ser indicado um condicionamento mieloablativo. ${ }^{1,18}$

\section{Fonte de células progenitoras}

O TCHP com células progenitoras periférica (TCHPP) por algumas sugestões de que oferece alguns benefícios e não há uma morbidade proibitiva, então, por estes motivos houve uma troca de fonte de células progenitoras da medula óssea para periféricas. ${ }^{18}$
As vantagens do TCHPP seria uma pega de neutrófilos e plaquetas mais rápidas, com diminuição deste tempo de aplasia pós-TCHP, portanto, com diminuição do risco de infecções e hemorragias. Em relação à doença do enxerto versus hospedeira aguda (DEvHa) entre grau 2 a 4 não há diferença, estatística, com a fonte de células progenitoras de medula óssea. Por outro lado, quando se compara grau 3 a 4 há uma maior incidência com o TCHPP. Há uma maior incidência do DEvH crônica quando realiza-se um TCHPP. ${ }^{20}$

Nas doença avançadas, ou seja, além da primeira FC, há uma vantagem em relação à sobrevida global, à sobrevida livre de doença, com diminuição da incidência da recidiva. ${ }^{18,20}$ ( Figura 5)

\section{Terapia de acompanhamento}

A figura 6 é um esquema realizado pela NCCN (Diretrizes de práticas clínicas em oncologia NCCN), para o período pós-TCHP.

Em resumo, o TCHP é altamente efetivo no tratamento da LMC, está indicado, na era dos inibidores da tirosina quinase, realmente, quando há mutação T315I. Deve-se pensar em TCHP quando há presença da mutação na região Ploop, se não há possibilidade de utilizar um inibidor da tirosina quinase de segunda geração. Ou quando há resistência ao imatinibe, se não há possibilidade de uso de um inibidor da tirosina quinase de segunda geração.

Em nosso meio, dependendo das possibilidades locais, ou seja, não havendo possibilidade de tratamento com imatinibe, o TCHP estaria indicado em primeira linha.

\section{Recomendações para o uso de inibidores de tirosinaquinase de segunda geração}

- Sempre devemos discutir a possibilidade do TCHP com os pacientes em qualquer fase da doença.

- Indicação em segunda linha doTCHP: deve ser após o tratamento em primeira linha com imatinibe.

- Indicação em terceira linha do TCHP: deve ser após o tratamento em segunda linha com outro inibidor da tirosinaquinase.

- A perda da resposta hematológica, citogenética e/ou molecular, estudar a presença de mutações. Se as mutações, forem resistente aos inibidores da tirosinaquinase, o TCHP está indicado.

- A progressão da doença para uma fase avançada é indicação de TCHP. 


\begin{tabular}{|c|c|c|c|c|c|c|}
\hline & \multicolumn{2}{|c|}{ Event/Patients } & \multicolumn{2}{|c|}{ Statistics } & \multirow{2}{*}{$\begin{array}{l}\text { OR and } 95 \% \mathrm{Cl} \\
\text { (PBSCT: BMT) }\end{array}$} & \multirow{2}{*}{$\begin{array}{l}\text { Odds Rdn. } \\
\text { (SD) }\end{array}$} \\
\hline & PBSCT & BMT & $(\mathrm{O}-\mathrm{E})$ & Var. & & \\
\hline Survival & $207 / 544$ & $234 / 563$ & -13.5 & 99.6 & $\rightarrow$ & $13 \%(9) ; 2 P=.2$ \\
\hline Disease-free survival & $223 / 544$ & $270 / 564$ & -24.0 & 109.9 & $\rightarrow$ & $20 \%(9) ; 2 \mathrm{P}=.02$ \\
\hline Relapse & $96 / 542$ & $132 / 558$ & -18.5 & 53.5 & - & $29 \%(12) ; 2 \mathrm{P}=.01$ \\
\hline Relapse mortality & $53 / 544$ & $79 / 563$ & -13.2 & 31.5 & $=$ & $34 \%(15) ; 2 \mathrm{P}=.02$ \\
\hline Non relapse mortality & $154 / 544$ & $155 / 583$ & -0.4 & 71.8 & & $1 \%(12) ; 2 \mathrm{P}=1.0$ \\
\hline aGVHD (II-IV) & $227 / 520$ & $213 / 541$ & 12.7 & 95.3 & - & $-14 \%(11) ; 2 P=.2$ \\
\hline cGVHD (extensive) & $189 / 483$ & $122 / 490$ & 38.8 & 61.1 & $\Rightarrow$ & $-89 \%(18) ; 2 \mathrm{P}<.0001$ \\
\hline Neutrophil engraftment & $516 / 530$ & $528 / 555$ & -98.4 & 83.2 & $\rightarrow$ & $69 \%(6) ; 2 \mathrm{P}<.0001$ \\
\hline \multirow[t]{3}{*}{ Platelet engraftment } & $471 / 532$ & $478 / 554$ & -86.7 & $\begin{array}{r}131.5 \\
\mathrm{~L}\end{array}$ & - & $\begin{array}{c}48 \%(6) ; 2 \mathrm{P}<.0001 \\
.\end{array}$ \\
\hline & & & & 0.1 & 1.0 & 10.0 \\
\hline & & & & & $\begin{array}{l}\text { PBSCT } \\
\text { Better }\end{array}$ & $\begin{array}{l}\text { MT } \\
\text { etter }\end{array}$ \\
\hline
\end{tabular}

Figura $5 .^{20}$ Sumário dos efeitos TCHPP versus TCHP

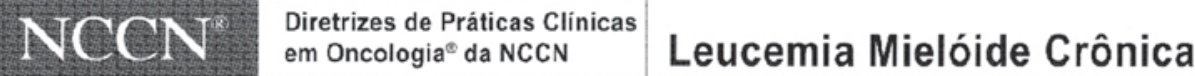

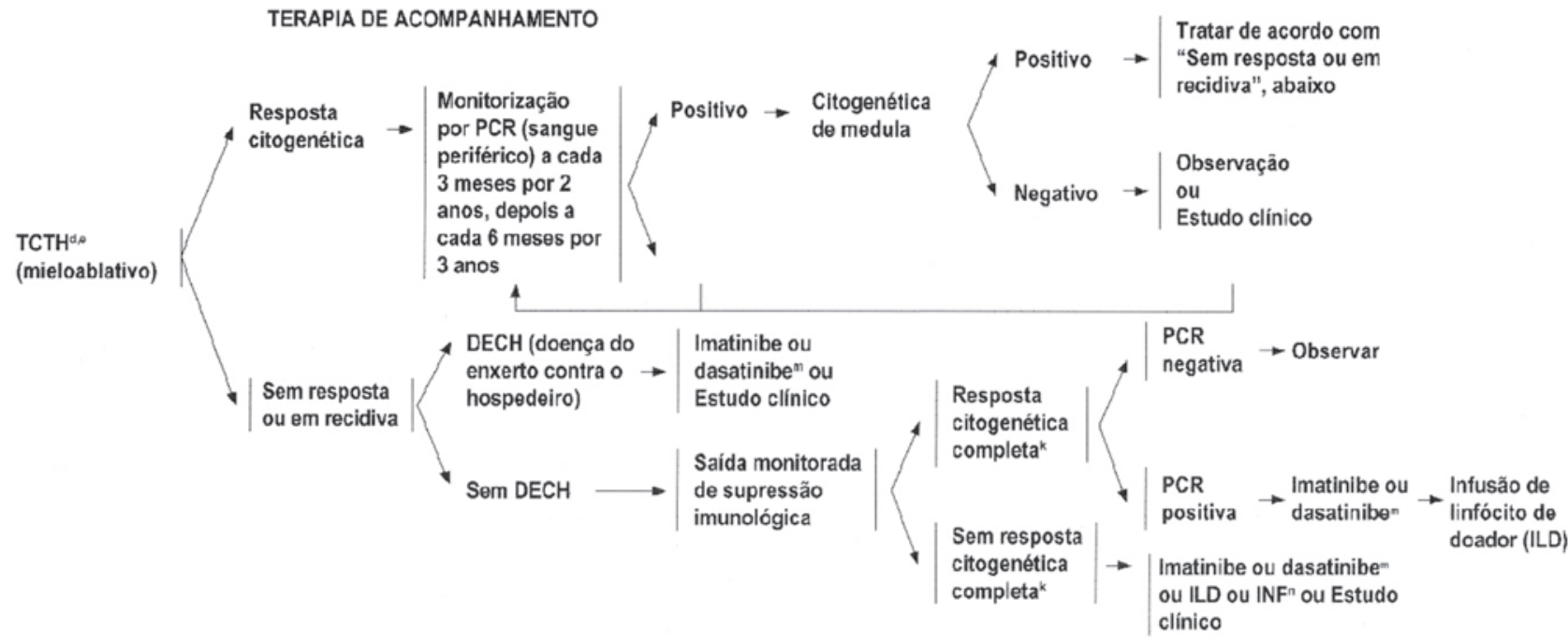

\footnotetext{
4 TCTH = transplante de célula-tronco hematopoiética. Refere-se a um transplante alogênico compativel com e sem parentesco. Devem ser realizados testes de HLA ao considerar TCTH.

- As indicações e os desfechos clínicos de resultados de transplante com e sem parentesco sắo: idade, tipo de doador e dependência do centro de transplante. $\mathrm{O}$ transplante não-mieloablativo está sendo pesquisado e deve ser realizado somente no contexto de estudo clinico.

" Existem dados para administração de imatinibe pós-transplante, mas nåo em pacientes nos quais imatinibe falhou anteriormente. Outros ITQ podem ser mais apropriados, embora não haja dados publicados que corroborem seu uso pós-transplante.
}

Figura 6. Fluxograma - Acompanhamento pós TCHP 


\begin{abstract}
Although the only curative therapy for chronic myeloid leukemia remains allogeneic stem cell transplantation (allo-SCT), the results of imatinib in newly diagnosed patients are sufficiently impressive to have displaced allo-SCT to second or third-line treatment. Patients now arrive at a decision for transplantation in a variety of disease situations: failing to achieve certain hematological, cytogenetic and molecular remission by some pre-determined timepoint, having lost a previous best response or due to progression to an advanced phase. The decision is also how to transplant. In this review article, the evidence supporting some of these decisions and current controversies are discussed. Rev. bras. hematol. hemoter. 2008; 30(Supl.1):41-46.
\end{abstract}

Key words: Chronic myeloid leukemia; bone marrow transplantation; hematopoietic progenitor.

\section{Referências Bibliográficas}

1. Goldman J. How I treat chronic myeloid leukemia in the imatinib era. Blood. 2007;110(8):2828-37.

2. Silver RT, Woolf SH, Hehlmann R, et al. An Evidence-Based Analysis of the Effect of Busulfan, Hydroxyurea, Interferon, and Allogeneic Bone Marrow Transplantation in Treating the Chronic Phase of Chronic Myeloid Leukemia: Developed for the American Society of Hematology. Blood. 1999;94(5):1517-36.

3. Goldman J. Management of chronic myeloid leukemia. Semin Hematol. 2003;40:1-103

4. Druker BJ, Talpaz M, Resta DJ, et al. Efficacy and safety of a specific inhibitor of the BCR-ABL tyrosine kinase in chronic myeloid leukemia. New Engl J Med. 2001;344:1031-37.

5. Gratwohl A, Baldomero H, HorisbergerB, et al. Current trends in hematopoietic stem cell transplantation in Europe. Accreditation Committee of the European Group for Blood and Marrow Transplantation (EBMT). Blood. 2002;100:2374-86.

6. Kantarjian H, Sawyers C, Hochhaus, et al. Hematologic and cytogenetic responses to imatinib mesylate in chronic myelogenous leukemia. New Engl J Med. 2002;346:645-52.

7. Gratwohl A, Brand R, Apperley J, et al. Allogeneic hematopoietic stem cell transplantation for chronic myeloid leukemia in Europe 2006: transplant activity, long-term data and current results. An analysis by the Chronic Leukemia Working Party of the European Group for Blood and Marrow Transplantation (EBMT). Haematologica. 2006;91:513-21.

8. Olavarria E, Apperley J. Managemente of patients with imatinib resistence. EHA. 2006;1(1):230-38.

9. Hughes TP, Kaeda J, Branford S, et al. Frequency of major molecular responses to imatinib or interferon alfa plus cytarabine in newly diagnosed chronic myeloid leukemia. New Engl J Med. 2003; 349: 1423-32.

10. Gratwohl A, Hermans J, Goldman JM, et al. Risk assessment for patients with chronic myeloid leukaemiabefore allogeneic blood or marrow transplantation. Lancet. 1998;352:1087-92.

11. van Rhee F, Szydlo RM, Hermans J, et al. Long term results after allogeneic bone marrow transplantation for chronic myelogenous leukemia in chronic phase: a report from the chronic leukemia working party of the European Group for Blood and Marrow Transplantation. Bone Marrow Transplant. 1997;20:553-60.
12. Devergie A, Apperley J, Labopin M, et al. European results of matched unrelated bone marrow transplantation for chronic myeloid leukemia: impact of class II matching. Bone Marrow Transplant. 1997;20:11-9.

13. Hansen JA, Gooley TA, Martin PJ, et al. Bone marrow transplants from unrelated donors for patients with chronic myeloid leukemia". N Engl J Med. 1998;338:962-68.

14. De Souza CA, Vigorito AC, Ruiz MA, et al. Validation of the EBMT risk score in chronic myeloid leukemia in Brazil and allogeneic transplant outcome. Haematologica. 2005;90:232-37.

15. Passweg JR, Walker I, Sobocinski KA, et al. Validation and extension of the EBMT Risk Score for patients with chronic myeloid leukaemia (CML) receiving allogeneic haematopoietic stem cell transplants. BJH. 2004;125:613-20.

16. Petersdorf EW, Anasetti C, Martin PJ, et al. Limits of HLA mismatching in unrelated hematopoietic cell transplantation. Blood. 2004.104:2976-80.

17. Guglielmi C, Arcese W, Dazzi F, et al. Donor lymphocyte infusion for relapsed chronic myelogenous leukemia: prognostic relevance of the initial cell dose. Blood. 2002;100:397-405.

18. Apperley J. Managing the patient with chronic myeloid leukemia through and after allogeneic stem cell transplantation. American Society of Hematology. 2006;226-232.

19. Gratwohl A, Baldomero H, Frauendorfer K, Urbano-Ispizua A. (2006). EBMT activity survey 2004 and changes in disease indication over the past 15 years. Bone Marrow Transplant. 2006; 37:1069-85

20. Stem Cell Trialists' Collaborative Group. Allogeneic peripheral blood stem-cell compared with bone marrow transplantation in the management of hematologic malignancies: An individual patient data meta-analysis of nine randomized trials. J Clin Oncol. 2005;23:5074-87.

O tema apresentado e o convite ao autor constam da pauta elaborada pelos co-editores, Professor Ricardo Pasquini e Professor Cármino Antonio de Souza.

Avaliação: Co-editores e um revisor externo. Publicado após revisão e concordância do editor. Conflito de interesse: não declarado.

Recebido: 15/02/2008

Aceito: 29/03/2008 